\title{
Preliminary concept of modern X-ray timing mission
}

\author{
Vadim Arefiev ${ }^{1}$ \\ Space Research Institute \\ Profsoyuznaya str. 84/32, Moscow, Russia \\ E-mail: arefiev@iki.rssi.ru

\section{Mikhail Pavlinsky} \\ Space Research Institute \\ Profsoyuznaya str. 84/32, Moscow, Russia \\ E-mail: pavlinsky@iki.rssi.ru

\section{Mikhail Revnivtsev} \\ Space Research Institute \\ Profsoyuznaya str. 84/32, Moscow, Russia \\ E-mail: revnivtsev@iki.rssi.ru
}

We present the concept of new X-ray timing mission proposed for Russian astrophysical program. The preliminary study includes the main scientific tasks for the X-ray timing mission, requirements for scientific payload and spacecraft. The mission will concentrate on study X-ray variability of bright accreting black holes and neutron stars. These objects can give unique information for both strong gravity and nuclear physics. The study of accretion flows variability mechanism is essential to successful interpretation of X-ray data and unambiguous determination of fundamental parameters of compact objects. The main instrument of the observatory is large area X-ray timing spectrometer.

Fast X-ray timing and spectroscopy at extreme count rates: Science with the HTRS on the International $X$-ray Observatory -HTRS 2011

Champéry, Switzerland

February 7-11 2011

$1 \quad$ Speaker 


\section{Introduction}

Variability of X-rays generated near accreting black holes or neutron stars holds information about matter and radiation under extreme conditions: strong gravity and magnetic fields, high temperature and pressure. Due to small size of stellar mass black holes and neutron stars (their radius less than 10-30 km) typical time variability scale of accreting matter is order of milliseconds or less. The variability of X-rays generated by accreting matter has the same time scale.

The observation of accreting black holes and neutron stars at X-rays would allow us to explore:

- the equation of state of super dense nuclear matter;

- the test of General relativity in the strong field mode;

- the matter behavior at high temperatures, pressures and strong magnetic fields (up to $10^{8}$ $\left.-10^{14} \mathrm{G}\right)$

- the mechanisms of X-ray generating in vicinity of compact objects.

The scientific problems mentioned above are fundamental physical questions of nuclear physics, gravitation theory and plasma physics.

Because of the variability (accompanied with related photon spectra) would become a key for solving of fundamental physical problems one would think about dedicated astrophysical timing X-ray observatory. We consider below the requirements for such new generation X-ray timing observatory, preliminary called as "X-ray microphone" [1].

\section{Mission science and experimental requirements}

\subsection{Timing science requirements}

Analysis of accreting black holes and neutron stars fast X-ray variability would allow undertaking:

1) the determination of high dense matter equation of state (neutron/quark stars) by measuring X-ray pulsation during the thermonuclear burning flashes on neutron stars surface, as well as study of period distribution of neutron stars.

2) the study of matter behavior under influence of strong gravitation fields of black holes and neutron stars with observation of X-ray QPOs and emission/absorption lines (most probable iron and oxygen lines).

3) the study of matter behavior under influence of high temperatures, pressure and magnetic fields with X-ray oscillation during magnetars bursts and with millisecond variability of X-ray generated in the pulsar accretion columns.

To solve these problems one need to increase the number of detected X-ray photons by order of magnitude in comparison with the current values achieved with RXTE - the only X-ray timing astrophysical observatory ever. 
Three conditions must be satisfied:

1) Maximize $X$-ray detector effective area up to $10-20 \mathrm{~m}^{2}$.

2) Optimize the detector energy band. The photon spectrum drops with photon energy (sometimes as $d N / d E \sim E^{-2}$, sometimes even faster) for the most interesting bright X-ray sources. One would require the lower energy range of the detector being at $1-2 \mathrm{keV}$ as minima.

One needs to mention several limitations for the value of high energy range. At first, absolutely necessary of having the upper energy limit well above the emission line region (near 6-7 keV). There are many problems to be resolved with high-precision observations of these emission lines in the supermassive black holes (AGN) spectra.

The proper interpretation of X-ray bursts parameters depends on which state (hard or soft) the X-ray burster occurs during the burst. It is important for precise determination $M / R$ ratio of neutron star and finding limitations on its equation of state. To find the spectral state properly one needs to measure photon spectra up to $20-30 \mathrm{keV}$. It will also dramatically simplified the interpretation of soft part of registered X-ray spectrum.

Therefore the upper energy limit of the X-ray detector should lay at $20-30 \mathrm{keV}$. The higher energy range even quite interesting for many astrophysical tasks is not so important for the Xray timing purposes.

3) The detector energy resolution near $6-7 \mathrm{keV}$ emission line region should be not worst than $5-10 \%$.

\subsection{Mission parameters}

Resulting requirements for the main experiment of "X-ray microphone" a new X-ray timing mission proposed for Russian astrophysical program [1] are summarized bellow:

Main instrument is Large Area X-ray Timing Spectrometer (LA XRTS)

Geometric detector area $>10 \mathrm{M}^{2}$

Timing resolution $<10^{-5} \mathrm{sec}$

Energy range 2-30 (1- 60) keV

Energy resolution600(300)eV@6 keV

Maximum count rate up to $10^{6} \mathrm{cnt} / \mathrm{s}$

FOV $\sim 1$ deg

Power consumption $<1500 \mathrm{Wt}$

The sketch of "X-ray microphone" is given on fig. 1. Green-yellow cubes are modules of LA XRTS - main experiment of the X-ray observatory.

The gray tube at the center is the X-ray grazing-incident mirrors telescope with focal Xray polarimeter. This experiment will make complimentary science, providing polarimetry measurements of bright X-ray binaries and AGNs. X-ray polarimetry is not only significant in its own right, but also provides important information about source geometry, which in turn helps with interpretation data from LA XRTS. 


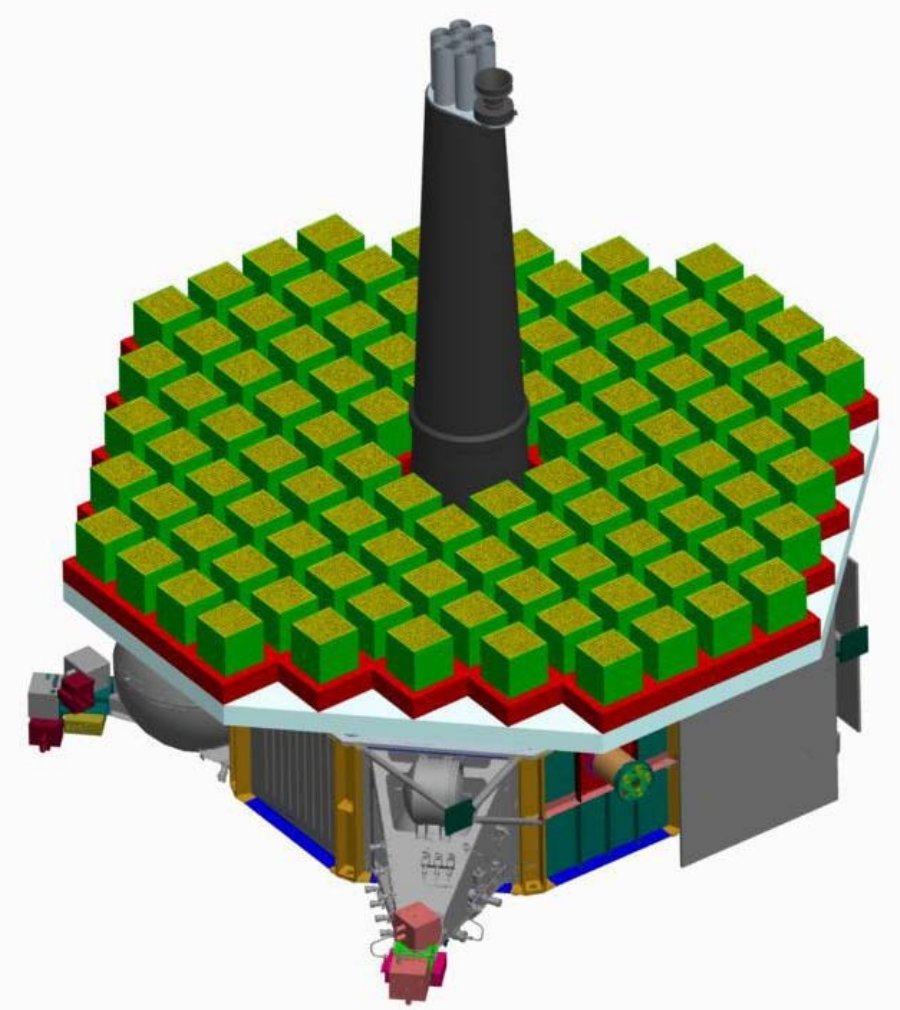

Fig. 1. Sketch of X-ray timing mission "X-ray microphone".

\section{LA XRTS detector and data handling system}

Those requirements (detector area $>10 \mathrm{~m}^{2}$ energy range $2-30(1-60) \mathrm{keV}$, energy resolution $600(300) \mathrm{eV}$ at $6 \mathrm{keV})$ are quite controversial and will require careful optimization of the X-ray detector design. In particular the hierarchical approach when detector is divided onto standard sections, section is divided on detector assembly, detector assembly is divided onto detector units consisting of elementarily detector is quite attractive (see fig.2). The hierarchical modular system will allow the wide dynamical range more than $10^{5}$. It is important for study of magnetar bursts as well as for observation of bright X-ray transients.

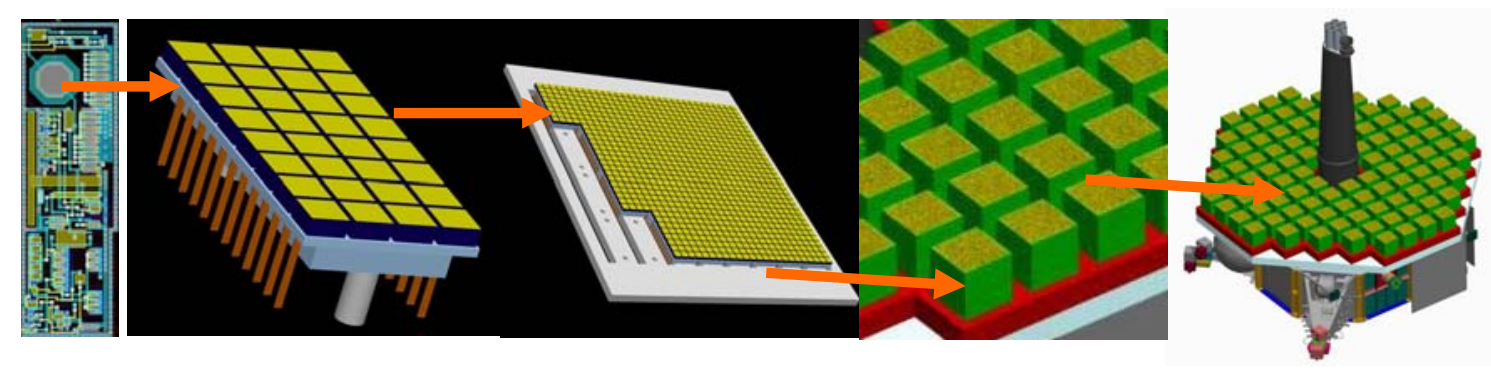

Fig.2. Modular detector design of LA XRTS 
In the such design the main parameters of the large area detector are defined by parameters of the smallest part - the elementary detectors. It is worth of noting that the requirements of low energy threshold at $1 \mathrm{keV}$ and energy resolution at $300 \mathrm{eV}$ at $6 \mathrm{keV}$ will lead naturally to elementary detector pixellization. The pixel size should be less than $1 \mathrm{sq} . \mathrm{mm}$. It will provide the detector spatial resolution with the similar value "for free". The spatial resolution even is not necessary for X-ray timing, might be very useful if one decide to implement this detector for wide-field $\mathrm{X}$-ray monitor.

The planned energy range (2-30 keV) and energy resolution $(600 \mathrm{eV})$ would allow to use the 525 micron pixilated Si detector connected with market-available ASIC. The desired energy range $(1-60 \mathrm{keV})$ and energy resolution $(300 \mathrm{eV})$ will require the development of low-noise, low-power consuming, radiation hard custom ASICs, to collect information from 256 - 1024 pixels of elementary Si detector. The preliminary design of a spectrometric channel of custom ASIC is under development.

The modular detector design will simplify the design of the data handling system, improves the reliability of the system. Data handling system must satisfy several criteria:

It should be able to collect and process up to $10^{6} \mathrm{cnt} / \mathrm{s}$

It should be scalable and easy to design, production and testing

It should have a high reliability, low power consumption

The number of most critical and most expensive components should be minimized

We have considered the tree-like structure, where the lowest level is related to collection data from individual ASICs, the next level collects data from several units, the next level collects data from integrated detector modules

\section{The satellite}

The "X-ray microphone" payload will be designed such way to be installed on Navigatortype bus (new development of Lavochkin Association). The first Navigator was successfully launched 20 January 2011 (Electro-L satellite). The following Navigators will be used for two major astrophysical projects: Radioastron and Spectrum-X-Gamma. In case "X-ray microphone" chosen orbit will be near Earth orbit (which is preferable from point of view detector background minimization), minor modification of Navigator would be required.

\section{References}

[1] V. Arefiev, X-ray microphone, in proceedings of HEA-2010, IKI, Moscow, 2010 